\title{
Human and organisational factors in planning and control
}

This special issue of Production Planning \& Control addresses the perspective of human and organisational factors in planning and control. This topic has been hindered during several years by the importance of economic performances in industry and is today of a high importance for managers as well as academicians.

The articles presented in this issue are selected from the 1st conference on Human and Organisational Factors in Planning and Scheduling (HOPS) in Lausanne, Switzerland, 8-9 September 2008. The conference was organised and hosted by the Swiss Federal Institute of Technology at Lausanne (EPFL) with the support of the University of Applied Sciences Northwestern Switzerland (FHNW) and the Eindhoven University of Technology (TUE). Over 50 contributions have been presented during the conference covering subjects such as behavioural analysis of planners and schedulers in industrial environments, integration of human knowledge and competence in planning and role of schedulers in global scheduling systems.

In his article, 'Order release in synchronous manufacturing', Riezebos identifies the relationship between human motivation and the working schedules with respect to stability issues. He designs heuristics based on insights from assembly system design and work load control to be used by employees within synchronous manufacturing systems.

In their article 'Contextual conditions influencing the scheduler's work at a sawmill', Karltun and Berglund analyse the contextual factors influencing the scheduling work in a Swedish sawmill. From this case study, they identify the contextual conditions that serve as means for the scheduler to plan production and thereby to control the technical system.

The article by von der Weth and Starker, entitled 'Integrating motivational and emotional factors in implementation strategies for new enterprise planning software' focuses on the role of motivation, emotion and knowledge in the success or failure of ERP implementation in industry. Based on this study, a strategy for ERP software integration in shop floor is proposed, on the basis of which two case studies are presented and discussed.

In their work entitled 'Visual scenario analysis: understanding human factors of planning in rail engineering', Schock et al. carry out a research work aiming at understanding the factors and settings that affect the performance and identify the human and organisational factors to be considered when planning work in rail engineering. Relying upon their findings, they develop a visual decision support tool to identify problems linked to worker safety and to the planning process.

The article by Marmier and Cheikhrouhou, 'Structuring and integrating human knowledge in demand forecasting: a judgemental adjustment approach', addresses the explicit and the implicit contextual human information and its usefulness in forecasting approaches. They propose a new socio-technical forecasting system where the human factors considered are associated to probable events that only experts/forecasters can identify, estimate and integrate in demand planning. Through integrating structured judgmental information, the system supports better decision making in forecasting demand and this is particularly shown in two implementations.

In their article, 'An empirical study on reducing planning instability in hierarchical planning systems', Moscoso et al. investigate empirically the mechanism of planning instabilities, where an erratic ordering and updating behaviour in response to changing workload levels could generate even larger variability in the workload levels and lead times. Their findings from a single case study of advanced planning system implementation in an aerospace enterprise suggest that planning structure and frequency significantly influence stability.

The Guest Editors would like to thank the authors for their interest and contributions to this special issue. Such high quality of the presented articles could not be reached without the help of members of the HOPS 
community and the journal Editorial Board that served as referees at different stages.

Naoufel Cheikhrouhou Head of the Operations Management team Ecole Polytechnique Fédérale de Lausanne Switzerland naoufel.cheikhrouhou@epfl.ch

François Marmier Assistant Professor Université de Toulouse - MINES ALBI, France fmarmier@gmail.com 